\title{
Cyclic plasticity at pores and inclusions in cast Al-Si alloys
}

\author{
Jinghong Fan ${ }^{\text {a,b,*, David L. McDowell }}{ }^{\text {c }}$, Mark F. Horstemeyer ${ }^{\mathrm{d}}$, Ken Gall ${ }^{\mathrm{e}}$ \\ ${ }^{a}$ Division of Mechanical Engineering, Alfred University, Alfred, NY 14802, USA \\ ${ }^{\mathrm{b}}$ Department of Engineering Mechanics, ChongQing University, 400044, China \\ ${ }^{\mathrm{c}}$ GWW School of Mechanical Engineering, Georgia Institute of Technology, GA 30332, USA \\ ${ }^{\mathrm{d}}$ Materials and Engineering Science Center, Sandia National Labs, Livermore, CA 94551, USA \\ ${ }^{\mathrm{e}}$ Department of Mechanical Engineering, University of Colorado at Boulder, CO 80309, USA
}

Received 23 July 2001; received in revised form 10 May 2002; accepted 18 May 2002

\begin{abstract}
Finite element analyses of micronotches including pores and silicon particles of an A356 aluminum alloy were performed to elucidate microstructure-property relations for fatigue crack incubation. Several important findings resulted. By varying the particle and pore size, spacing, aspect ratio, and clustering, the relative microstructural differences were quantified related to micronotch root cyclic plasticity. Results from realistic two-dimensional microstructures showed that minimal microstructure-scale cyclic plasticity corresponds well to the measured fatigue strength at $10^{7}$ cycles for low porosity A356 aluminum alloy specimens. "Realistic" and idealized particles/pores simulations were used to formulate a local Coffin-Manson type law for crack incubation.
\end{abstract}

(c) 2002 Elsevier Science Ltd. All rights reserved.

Keywords: Fatigue; Cast alloys; Computational micromechanics; Particle effects; Pore effects

\section{Introduction}

The mechanical, physical, and casting properties of cast aluminum alloys such as A356-T6 make them attractive for use in cost-effective, lightweight engineering components. However, to successfully use such an alloy in components intended for long life applications it is necessary to understand its fatigue resistance. A356-T6 Al alloy is a highly heterogeneous material with several dominant types of inclusions that govern fatigue resistance. We use the term inclusion as distinctly different from a crack, as the former occurs naturally as a product of the casting process, while the latter is usually a result of applied loading.

In order to capture the microstructure-dependent fatigue properties, a microstructure-based fatigue model has been developed [1]. In this model, we decomposed the fatigue life into four stages. First, we modeled the incubation stage of fatigue life, the number of cycles required to form a small crack and propagate it within the influence of the micronotch root field. The second stage is the microstructurally

\footnotetext{
${ }^{*}$ Corresponding author. Present address: Division of Mechanical Engineering, Alfred University, Alfred, NY 14802, USA. Tel.: +1607-871-3781/2100; fax: +1-607-871-2114.

E-mail address: fanjing@alfred.edu (J. Fan).
} 
small crack (MSC) growth regime in which the crack length is less than several dendrite cell sizes (DCS). The crack then grows until it reaches a physically small crack (PSC) stage, which corresponds to a transition from MSC status to that of a dominant, long crack. For A356-T6 Al alloy, the DCS is typically on the order of $20-100 \mu \mathrm{m}$, and the PSC regime may conservatively extend for crack lengths up to $300-800 \mu \mathrm{m}$. It has been noted in the literature that $50-90 \%$ of the life is spent in these three stages [2].

Many researchers have combined these three stages, culminating in a PSC, into a single stage termed "crack initiation." We separate these stages, because they differ physically and in terms of appropriate models. Our use of the term "fatigue crack incubation" refers primarily to the quiescent period of crack formation at a notch root that is commonly referred to as fatigue crack nucleation, i.e., the number of cycles at which a crack should first form with length on the order of the scale of the notch root cyclic plastic zone. This definition of incubation implicitly includes growth of very small cracks from the pertinent micronotch (typically a distance of about $10-20 \%$ of the notch root radius), which is a scale on the order of microns for many of the inclusions considered here. Growth of microstructurally small cracks beyond this point is modeled explicitly with a suitable law. The term crack "initiation" as typically used pertains to the formation and growth of MSCs. Microstructure-based fatigue models are potentially useful for several different inclusion types which include oxides, pores, and second phase particles. We should mention that although we focus on a cast A356-T6 aluminum alloy, other materials and alloys could be analyzed with this same type of modeling approach.

Of considerable relevance to any microstructure-based fatigue model for cast alloys is the driving force for fatigue crack formation as a function of the dendrite cell size, sizes and morphologies of pores and particles, distance to a free surface, and applied stress level. With such a myriad of heterogeneities and factors that contribute to fatigue resistance, it is easily understood why separation of these attributes is difficult from an experimental viewpoint. Due to the large range of length scales and types of inclusions which may be present in a given casting, two important observations are worth mentioning. First, the fatigue life for a given loading condition may vary substantially over a range of specimens obtained from the same casting (a factor of ten variation is not uncommon), even under low cycle fatigue conditions, quite distinct from wrought alloys. Second, experiments can lead to ambiguous conclusions regarding controlling mechanisms of fatigue crack formation and propagation through the microstructure. This is because cracks can form at unknown locations within the bulk and propagate undetected by surface inspection through much of the fatigue life, especially under high cycle fatigue (HCF) conditions where the nominal response is linear elastic. It is difficult to perform enough experiments to obtain sufficient observations of fatigue crack formation and early propagation through the microstructure for each inclusion type to formulate truly interpolative or extrapolative empirical fatigue life estimation schemes that contain microstructure parameters.

On the other hand, microstructural elastoplastic finite element analysis for cyclic loading conditions can offer a great deal of information to help clarify our understanding of microstructure-property relations and to provide necessary information for the development of microstructure-property fatigue models. Our previous work [3] on computational micromechanics analysis of fatigue crack propagation in a casting aluminum alloy concentrated on small fatigue crack tip behavior related to the MSC stage. In that paper, we used the crack tip displacement as the driving force for small crack propagation. In this paper we concentrate on the first stage, crack incubation. Since the maximum range of the local cyclic plastic shear strain within the microstructure is a primary driving force for fatigue crack formation, we focus on microstructure scale plasticity using finite element analyses of both idealized geometries and more realistic representation of actual microstructure/inclusion morphologies obtained from optical images. In particular, we examine the effects of applied stress amplitude and microstructure on the maximum plastic shear strain range. Effects of sizes and morphologies of particles and pores on fatigue crack incubation life are evaluated based on local Coffin-Manson type criteria. These analyses are based on nonlinear kinematic hardening constitutive laws [4] with experimentally determined material constants for both the $\mathrm{Al}-1 \% \mathrm{Si}$ dendrite matrix region and the overall A356-T6 aluminum alloy under cyclic loading. 


\section{Fatigue crack incubation}

\subsection{Experimental observations}

Plumtree and Schafer investigated the initiation and early growth of fatigue cracks in strain cycled squeeze-cast $\mathrm{Al}-7 \% \mathrm{Si}$ alloy using single-stage replication techniques [5]. They reported that fatigue cracks initiated at the silicon particles in the eutectic region, generally at triple points, and then propagated through the eutectic regions. Lee et al. investigated fatigue failure characteristics and mechanisms in $\mathrm{Al}-\mathrm{Si}-$ $\mathrm{Mg}$ eutectic casting alloys with a composition just beyond the eutectic point in the Al-Si equilibrium phase diagram, i.e., approximately $\mathrm{Al}-12 \mathrm{wt} . \% \mathrm{Si}$ and $0.35 \mathrm{wt} . \% \mathrm{Mg}[6]$. The higher silicon content of $12 \%$ for their alloy compared to 7\% Si for A356-T6 perhaps explains their observation of Si particle-induced fatigue crack formation. They reported that during cyclic plastic deformation, formation of cracks occurred by particle-matrix interface separation and/or by particle cracking. The interfacial strength between particle and matrix is a primary factor controlling crack formation. They also reported that other factors that may contribute to the void/microcrack initiation process include: size, volume fraction, shape, strength, location, and orientation of particles. They found that the presence of large and irregularly shaped Si particles with large aspect ratios facilitates and accelerates crack formation due to the additional stress concentration effect of particles.

Clearly, to understand the mechanisms of fatigue crack incubation at or near particles, we must quantify particle mechanical properties and the relationships between particle geometry and stress/plastic strain concentration around the particles. Silicon particles are much stiffer than the surrounding aluminum rich matrix and deform elastically. The matrix is an elastic-plastic material with a low work hardening rate. When the particle cracks or debonds from the matrix, stresses are transferred to adjacent regions of the microstructure. When this occurs, the matrix is subject to intense plastic shear strain localization. Finite element results show that particle debonding gives a local intensification of plastic strain within the Al$1 \% \mathrm{Si}$ matrix that is significantly larger than that due to particle fracture [7]. The results also show that when several particles within a cluster debond, the spacing between adjacent particles becomes a dominant effect due to the large local stress/plastic strain intensification in the surrounding $\mathrm{Al}-1 \% \mathrm{Si}$ matrix. From a physical point of view, these phenomena are closely related to the intense dislocation activity in the matrix, giving rise to persistent slip bands (PSBs) near the debonded and fractured particles. In fact, previous metallurgical investigations have shown that the fracture of silicon particles embedded in an aluminum matrix is linked to dislocation motion in the aluminum matrix and dislocation pile-up at the interface between matrix and particles [8,9].

Large-scale oxide films are highly prone to forming large fatigue cracks [10]. Locations of crack formation have been traced to aluminum oxide inclusions in several studies [11]. The critical oxide inclusion is usually relatively close to the specimen surface [11-13].

Couper et al. [14] cited examples of fatigue cracks that started from casting pores. The microporosity levels engendered by entrapped hydrogen gas in the melt, which grow in concert with the dendrite cell size (secondary dendrite arm spacing) as the microstructure coarsens with solidification, also contribute significantly to the localization of cyclic plastic shear strain in the interdendritic regions under fatigue loading. Experimental results and theoretical analyses by Ting and Lawrence showed that there is an intensification of cyclic plastic strain (slip) between pores and the free surface under cyclic loading [12]. This intense cyclic plastic strain concentration/slip between pores and the free surface under cyclic loading was calculated in the present work; Fig. 1 shows the contours of maximum plastic shear strain range for a completely reversed $\left(R_{\varepsilon}=\varepsilon_{\min } / \varepsilon_{\max }=-1\right)$ applied tension-compression strain amplitude of $\varepsilon_{\mathrm{a}}=0.2 \%$. The slip band is nearly $45^{\circ}$ to the free surface. The location of the maximum plastic shear strain occurs near the tip of the ellipse that is nearest to the free surface. This intensification leads to formation of a fatigue crack on pore surface which is near the free surface by persistent slip banding. For internal pores, both gas pores and 


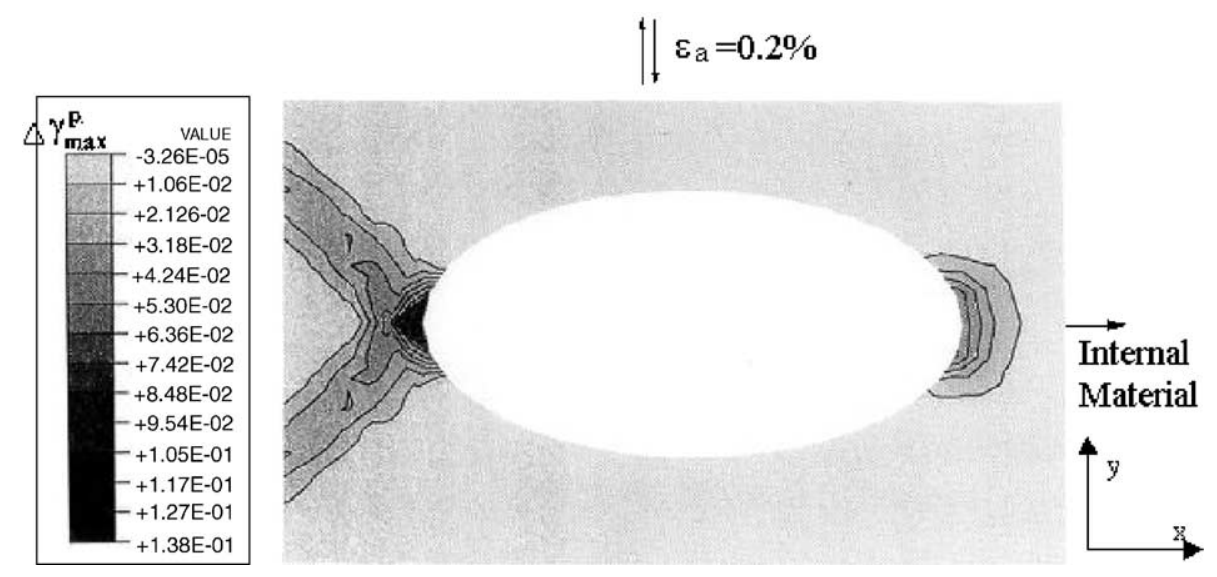

Fig. 1. Contours of the maximum plastic shear strain range around a pore near a free surface $\left(R_{\varepsilon}=-1, D_{\max } / D_{\min }=2, D_{\max }=40 \mu \mathrm{m}\right.$, $\varepsilon_{\mathrm{a}}=0.2 \%$. Boundary conditions: uniform cyclic displacement along the $y$-direction at the top side, simple support at the right side with $u_{x}=0$, simple support at the bottom side with $u_{y}=0$, and free at the left side).

shrinkage pores affect the local intensification of cyclic plastic strain and fatigue/fracture resistance of the alloy. Large shrinkage pores are especially potent in degrading the fatigue resistance; they serve as sites for formation of particularly large cracks that propagate to premature failure.

Couper et al. [14] reported a direct experimental observation of fatigue crack formation that was caused by PSBs without involving defect-intensified mechanisms. They reported that "...fine parallel surface cracks or PSBs were seen in favorably oriented grains, particularly close to the grain boundaries. Sectioning of samples also confirmed that these cracks were not associated with subsurface porosity." Fatigue crack incubation in cast aluminum alloys with low porosity is relevant since advances in casting technology have continuously improved control of the largest pore size and have minimized the introduction of oxide films into the pour. Couper et al. [14] also mentioned an observation that "reduction in pore size below approximately $15 \mu \mathrm{m}$ would not further improve the fatigue life." They introduced the concept of a critical pore size below which further reduction of pore size did not increase fatigue life. They suggested using notched specimens for fatigue analysis to exclude the pore effects and said that "until such an analysis is done it is not possible to fully specify a minimum pore size below which classical crack initiation will determine the fatigue life." In their work, the critical pore size was discussed in the context of competitive mechanisms of pore-induced fatigue crack incubation versus classical fatigue crack formation in a matrix without pores. This concept can be extended to the critical pore size under which other mechanisms such as particle debonding or cracking adopt a dominant role for crack incubation compared to pore-controlled mechanisms.

\subsection{Mechanisms and criteria for fatigue crack incubation}

From the above-mentioned observations, we assert that the mechanism of fatigue crack incubation is intense cyclic plastic shear strain. The maximum range of cyclic plastic shear strain, $\Delta \gamma_{\max }^{\mathrm{p} *}$, can be regarded as a continuum-based driving force for fatigue crack formation. This explains why fatigue cracks frequently form near the free surface in castings, at triple points of particle clusters, or near debonded or fractured particles. This viewpoint is consistent with the minimum energy formulation for fatigue crack nucleation in a multiple slip band proposed by Venkataraman et al. $[15,16]$. In their multiple slip model, multiple parallel 
slip bands are considered. Each slip band is modeled as an accumulation of edge dipoles. The dislocation dipole density within the slip bands continuously increases with fatigue cycling. The strain energy density within the slip bands consequently builds up with cycling until a critical number of cycles when it becomes energetically favorable to nucleate a microcrack within one of the slip bands. Venkataraman et al. [15,16] showed that there was an analogy between this criterion for crack nucleation and other criteria that are based on the accumulation of a certain critical amount of damage. A generalized Coffin-Manson law for crack nucleation was then proposed which was an extension of the work of Kato et al. [17]. Based on this idea, a simple local form of the Coffin-Manson law is used in this work to correlate the number of cycles, $N_{\text {inc }}$ for incubation of a fatigue crack with the maximum range of cyclic plastic shear strain, $\Delta \gamma_{\max }^{\mathrm{p} *}$, in the Alrich matrix material. This law is expressed as

$$
\beta=\frac{\Delta \gamma_{\max }^{\mathrm{p} *}}{2}=C N_{\mathrm{inc}}^{\alpha}
$$

where $\Delta \gamma_{\max }^{\mathrm{p} *}$ is the micronotch root maximum plastic shear strain range in the Al-rich matrix, and $C$ and $\alpha$ are constants. In general, $\Delta \gamma_{\max }^{\mathrm{p} *}$ significantly exceeds the average macroscopic plastic shear strain. The incubation life, $N_{\text {inc }}$, is related to the magnitude of cyclic plasticity in the vicinity of microstructural notches or casting inclusions.

Another incubation criterion is adopted for the general case where the normal stress perpendicular to the slip plane plays an important role in fatigue crack formation and small crack growth over length scales much larger than average interparticle spacing. Since small crack propagation has been found to follow the interface of debonded particles and matrix [11], it is sensible to modify the criterion to include normal stress effects. Following Refs. [18,19], a shear-based criterion with normal stress dependence to the maximum shear plane is given by

$$
N_{\text {inc }}^{\alpha^{\prime}} \Delta \Gamma=C^{\prime}
$$

where

$$
\Delta \Gamma=\Delta \gamma_{\max }^{\mathrm{p}}\left[1+k \frac{\sigma_{\mathrm{n}}^{\max }}{\sigma_{\mathrm{y}}}\right]
$$

and $\sigma_{\mathrm{n}}^{\max }$ is the normal stress perpendicular to the slip plane, and $\sigma_{\mathrm{y}}$ is the cyclic yield strength. Constants $\alpha$ and $C$ and $k, \alpha^{\prime}$ and $C^{\prime}$ are material dependent. Constants $\alpha$ and $C$ have characteristic values for the Al-rich matrix, while $k, \alpha^{\prime}$ and $C^{\prime}$ pertain to the A356-T6 Al alloy. These constants can be respectively characterized by experiments on specimens of Al-1\%Si matrix and A356-T6 alloy under low cycle fatigue loading conditions where the Coffin-Manson law is valid. In this work, the specimens that we used for determining these constants had approximately the same composition and microstructures as the specimens that were used for determining the cyclic stress-strain curves (see Figs. 2 and 3). These curves were used for determining material constants of the constitutive laws. Using the same materials for determining material constants for both constitutive laws and crack incubation criteria makes the analysis consistent. For Al$1 \% \mathrm{Si}$ matrix we found $\alpha=-0.5, C=0.021$; for A356-T6 Al alloy $\alpha^{\prime}=0.546, C^{\prime}=0.0622$, and $k=0.95$. In this work, Eq. (1) is used for crack incubation near particle clusters, where crack formation occurs in the Al-rich matrix only. Eq. (2) is used for cases of pore clusters where cracks are considered to be incubated (formed and propagated) within the notch root cyclic plastic zone, which may extend over a number of silicon particles. 


\section{(a) Experimental Curve}

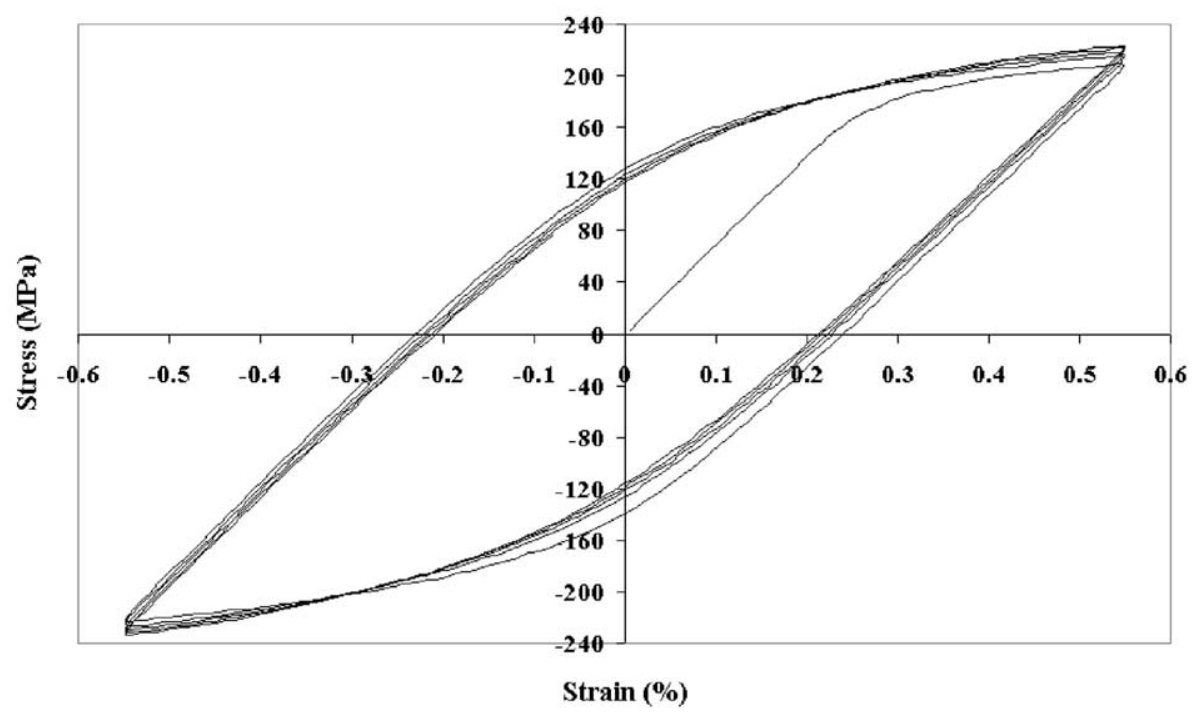

(b) Simulation

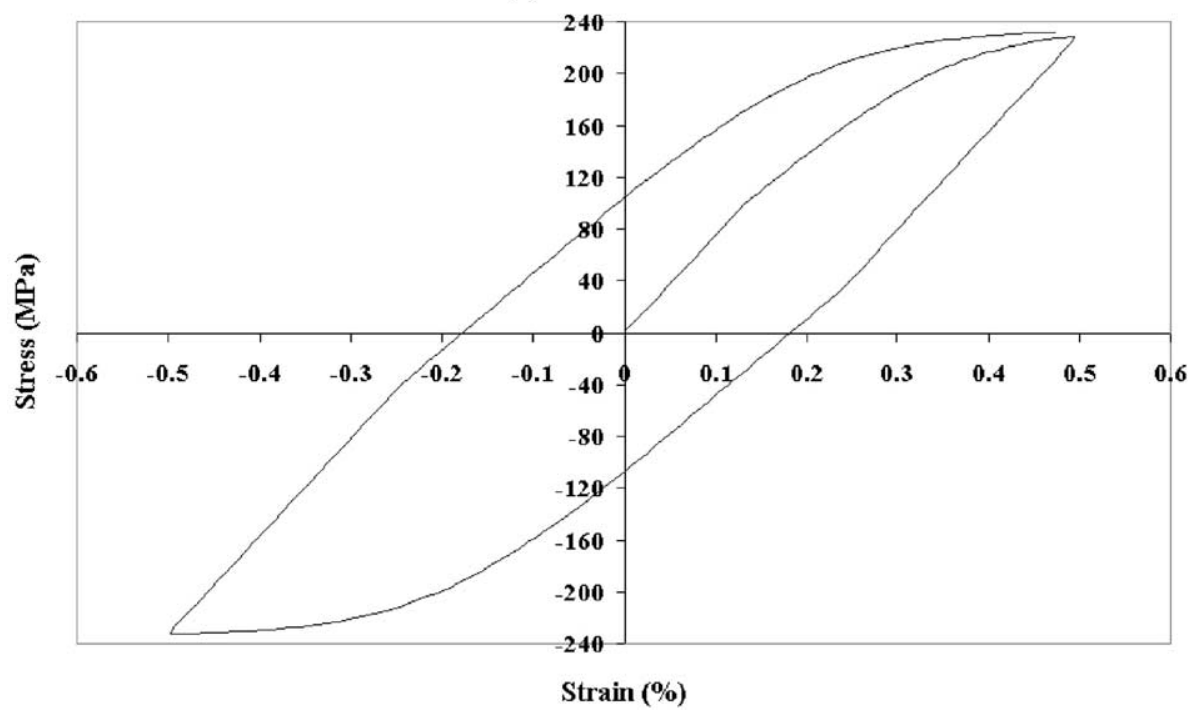

Fig. 2. A comparison of stress/strain curve for the Al-1\%Si matrix showing the cyclic experimental data and the simulation curve using the obtained material constants: (a) experimental curve and (b) simulation.

\section{Methodology}

To apply criteria (1) and (2) for determining the number of cycles required for crack incubation, $N_{\text {inc }}$, it is necessary to determine the maximum range of cyclic plastic shear strain, $\Delta \gamma_{\max }^{\mathrm{p} *}$. Due to the complexity of the geometry and morphology of microstructure in a cast alloy, it is difficult to analytically and experimentally estimate this value. The most fruitful approach is to carry out a microstructural finite element analysis. Before discussing the finite element analysis, it is necessary to address the concept of averaging 
(a) Experimental Curve

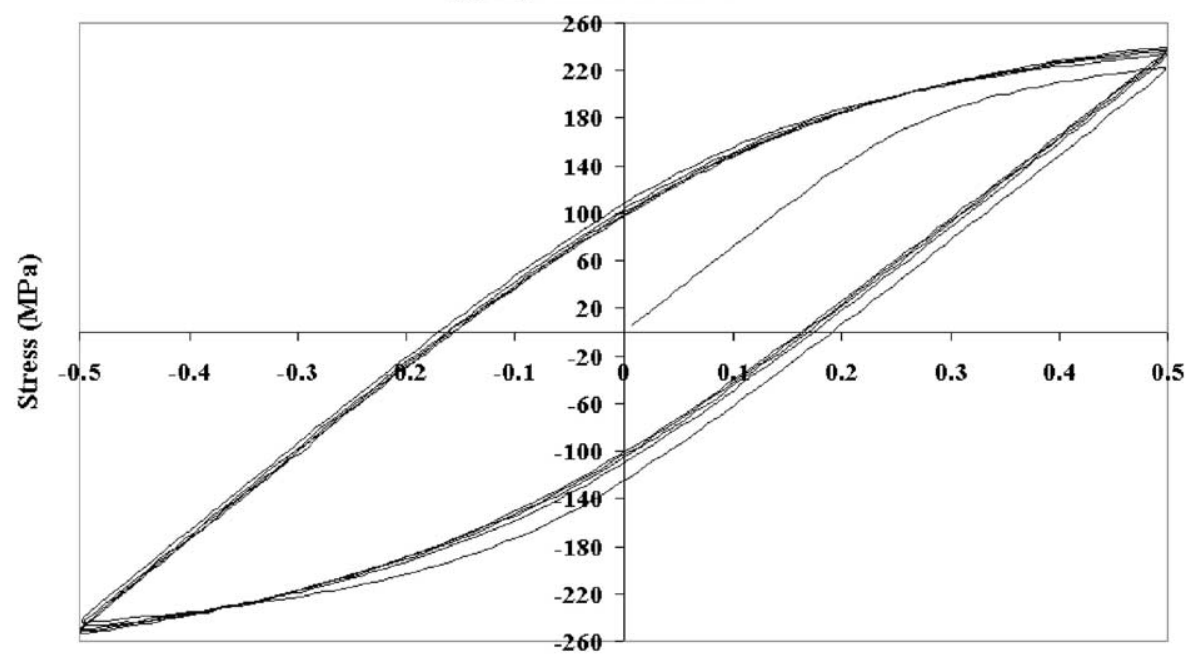

Strain $(\%)$

(b) Simulation

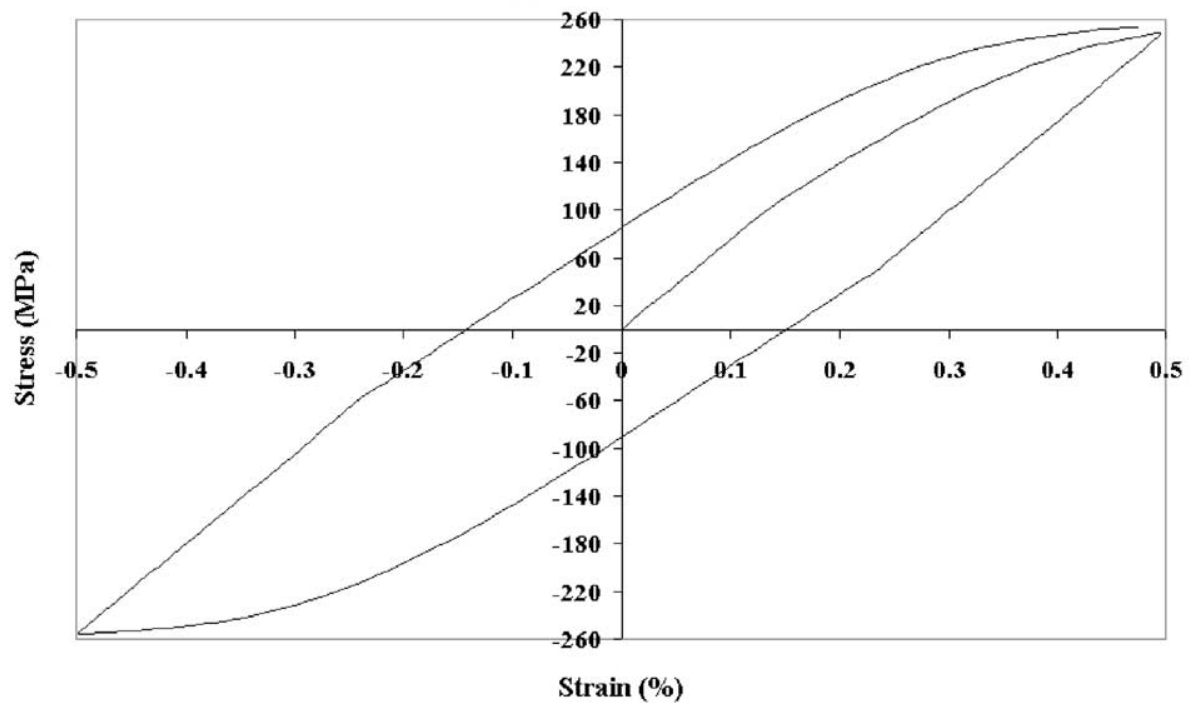

Fig. 3. A comparison of stress/strain curve for A356-T6 Al alloy showing the cyclic experimental data and the simulation curve using the obtained material constants: (a) experimental curve and (b) simulation.

over a representative volume to obtain representative values of $\Delta \gamma_{\max }^{\mathrm{p} *}$. It is recognized that finite element analyses that use local continuum field theory may exhibit mesh dependence of solutions depending upon the assumed constitutive relationships and geometry. Physically, however, cyclic plastic deformation cannot exhibit arbitrarily high gradients in the limit as mesh size decreases. Hence, in using local constitutive relations we must set a limit on the finest length scale over which plastic strains are effectively averaged, i.e., the element size. For example, near the tip of a micronotch or crack in an actual wrought polycrystalline alloy, slip within each grain is much more uniform than would be predicted based on the assumption of 
material homogeneity, i.e., neglecting grain structure, simply because gradients of plastic deformation are accommodated and spread over finite distances by dislocation glide until barriers such as grain boundaries are encountered. In the case of fatigue crack formation, PSBs form within the interior of surface grains. Upon crack formation, energy is released over a segment of the PSB ranging from about half a micron to a few microns $[15,16]$. In other words, any parameter associated with the damage process zone of significant cyclic plastic strain must be subjected to a nonlocal averaging procedure. The size of a low order element may be used to define the scale of the domain over which stress and plastic strain are averaged. Finer elements may lead to increasingly localized and intensified cyclic plasticity with mesh refinement, a physically untenable result. In this study, the minimum micronotch root element sizes are about $0.5 \mu \mathrm{m}$ for triangular elements and $1 \mu \mathrm{m}$ for four-node isoparametric element at the notch roots of silicon particles. These are also the sizes that we used for meshes along the propagation path of MSCs within or near particle clusters in Ref. [3]. For crack incubation within or near pore clusters, the minimum notch root element size is larger in a manner commensurate with the significant increase in pore size relative to particles.

This work defines the "average" $\Delta \gamma_{\max }^{\mathrm{p} *}$ values as those corresponding to the nearest Gaussian point of the element at the micronotch root, except otherwise stated. For a triangular element, it is defined at the central point of the corresponding element. Taking the minimum element size, $0.5 \mu \mathrm{m}$, of a triangular element as an example, the $\Delta \gamma_{\max }^{\mathrm{p} *}$ value was effectively averaged over a volume of about $0.0625 \mu \mathrm{m}^{3}$. The equilibrium interatomic spacing of aluminum atoms is about $0.143 \mathrm{~nm} \mathrm{[20].} \mathrm{This} \mathrm{results} \mathrm{in} \mathrm{about} 4 \times 10^{11}$ atoms in the volume for averaging with respect to crack formation processes. The minimum averaging distance taken in this work is consistent with other results $[15,16]$ in which the estimated initiation size of a fatigue crack is about $0.5 \mu \mathrm{m}$. Both the effective medium (A356-T6) and the matrix ( $\mathrm{Al}-1 \% \mathrm{Si}$ ) are assumed to be linear elastic and plastically incompressible. The particles are assumed to be linear elastic. The constitutive behavior of both the matrix and effective medium is described by a nonlinear kinematic hardening law [4]. A rate-independent flow rule is employed, with the yield surface and kinematic hardening law given by

$$
\begin{aligned}
& \frac{3}{2}\left(S_{i j}-\alpha_{i j}\right)\left(S_{i j}-\alpha_{i j}\right)-\rho^{2}=0 \\
& \mathrm{~d} \alpha_{i j}=\sum_{r=1}^{n} \mathrm{~d} \alpha_{i j}^{(r)} \\
& \mathrm{d} \alpha_{i j}^{(r)}=C^{(r)}\left[b^{(r)} n_{i j}-\alpha_{i j}^{(r)}\right] \mathrm{d} p
\end{aligned}
$$

where $S_{i j}$ is the deviatoric stress, $\rho$ an isotropic hardening parameter, $\alpha_{i j}$ is the back stress related to kinematic hardening, $n_{i j}$ a unit vector, and $\mathrm{d} p$ is the increment of cumulative plastic strain. The latter two are defined as

$$
\begin{gathered}
n_{i j}=\frac{S_{i j}-\alpha_{i j}}{\left\|S_{i j}-\alpha_{i j}\right\|} \\
\mathrm{d} p=\sqrt{\mathrm{d} \varepsilon_{i j}^{\mathrm{p}} \mathrm{d} \varepsilon_{i j}^{\mathrm{p}}}
\end{gathered}
$$

Since the cyclic hardening rate saturates rapidly, the effect of the short term transient process from initial cycling to steady state on fatigue life is minimal. Thus, isotropic hardening is not considered and the radius, $\rho$, of the yield surface is therefore taken as a constant. Two terms $(n=2)$ in Eq. (3) are sufficient to describe the completely reversed cyclic hysteresis loops. In this case, there are only five material constants for the cyclic response of each material. They are $C^{(r)}, b^{(r)}(r=1,2)$ and $\rho$. To determine these constants, two completely reversed cyclic stress-strain tests were conducted (i.e., $R_{\varepsilon}=-1$ ). The first one is the cyclic stressstrain curve of the A356-T6 alloy that corresponds to the effective medium, and the other is for Al- $1 \% \mathrm{Si}$ (the Al-rich matrix). Figs. 2 and 3 show the comparison of the cyclic stress-strain curves between the 
experimental data and the simulated curves, respectively, for A1-1\%Si matrix and A356-T6 Al alloy, using these material constants. The A356-T6 specimens saturated to steady state quickly, after only about 10-15 cycles. Therefore, only the first several cycles were used to determine material constants. The satisfactory agreement obtained using only five constants for a description of the nonlinear behavior is essential to guaranteeing the accuracy of the calculation as well as to maintaining a reasonable computation time. This constitutive law was embedded into a UMAT, a user-defined subroutine in the ABAQUS finite element code [21].

All calculations, including those for the so-called "realistic" microstructures, are conducted under plane strain conditions. Given this idealization, extension of the results to general three-dimensional (3D) microstructures should be viewed with caution. Fig. 4 shows definitions of the notations used for the numerical analysis of both particles and pores. This figure also shows the arrangement of four particles (or pores) in most calculations of this work to investigate the interaction effects between particles (or pores). All particles and pores are assumed to be two-dimensional (2D) ellipses, with $D_{\max }$ and $D_{\min }$ as the major and minor axes, respectively. The average particle or pore size is defined by $D=\left(D_{\max }+D_{\min }\right) / 2$, and their aspect ratio is defined as $D_{\max } / D_{\min }$. In the parametric studies, except otherwise stated the bottom boundary was pin supported to allow motion only along the $x$-direction. A cyclic vertical displacement was prescribed at the top boundary. For the left boundary, there were two cases; one was traction free, while the other was allowed to move only along the $y$-direction. The former case was used for investigating pores near a free surface and the latter case was used for effects of particle/pore clusters with a symmetric boundary to introduce particle/pore interaction effects. For cases involving complicated microstructures that require significant CPU time, the area of the analysis was divided into two domains, an outer domain of effective homogeneous medium and an inner domain that comprised particles and matrix [3]. The outer domain (or the boundary area) was at least two times the size of the inner domain. Properties of A356-T6 Al alloy were used as an effective medium. For the idealized geometries, four-node isoparametric elements were used. For the realistic microstructure, three-node triangular elements were used to make the mesh near the boundary of particles/pores smooth. In some cases, mixed meshes were also used. Within the effective medium, fournode isoparametric elements were used. All loading was displacement controlled, with displacements applied uniformly along the external boundaries.

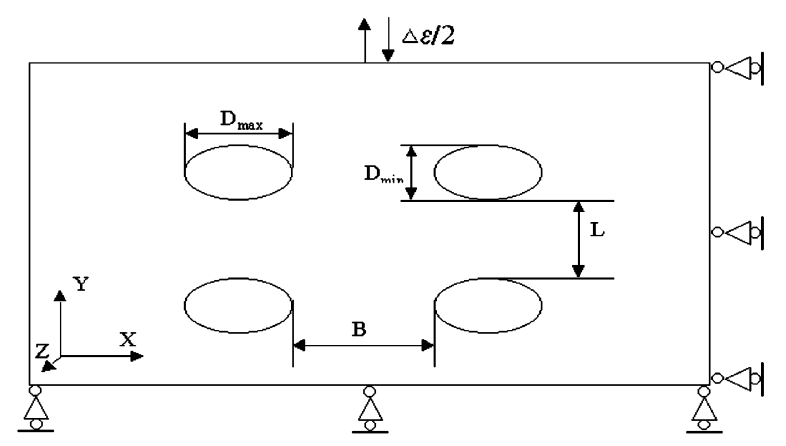

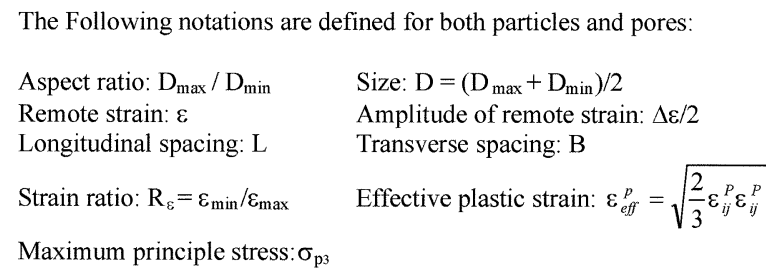

Fig. 4. Definitions of symbols and notations for particle and pore clusters. 


\section{Experiments and microstructure simulations for microstructures with extremely low porosity}

In this work, use of the term "realistic" microstructure characterizes 2D images obtained from specimens using optical image analysis. This image analysis offers the position, the size, and shape of pores/particles in the material, with the idealization that ellipses or polygons are used to represent pores and particles. While the so-called realistic microstructure and corresponding 2D computational analyses differ from 3D microstructure analyses, simulation of realistic microstructure distributions can offer useful information regarding trends of the structure-fatigue property relations.

In the actual as-cast material, residual stresses may be present due to differences in thermal expansion coefficients between matrix and particle, even following heat treatment. However, this paper will not address the interplay between residual stress and cyclic plastic deformation in the microstructure, with the principal objective to establish first order understanding of structure-fatigue property relations. While this is a simplified approach, it is effective in getting the trends or relative importance of different parameters because all the calculations are based on the same reference state of geometry and residual stress state.

\subsection{Results of multiaxial fatigue testing}

The composition of multiaxial fatigue specimens used in this work were the same as the specimens used in Ref. [22]. All the specimens including those used in [22] were fabricated using the same technique and were obtained from the same source of a high-grade A356.2 ingot. The cast alloy contained strontium-modified silicon particles ( $6 \%$ volume fraction) and an extremely low overall volume fraction of porosity $(<0.01 \%)$. SEM micrographs [22] revealed very fine microstructure of these specimens. Detailed microstructure characterization for a specimen with a long life showed that fatigue cracks formed at second phase inclusions such as oxide films or in the vicinity of particles rather than at casting pores. The results of several completely reversed multiaxial fatigue tests for the A356-T6 Al alloy are shown in Fig. 5. In this figure, the effective strain amplitude is defined as the von Mises form

$$
\varepsilon_{\mathrm{eff}}^{a}=\sqrt{\left(\frac{\Delta \varepsilon}{2}\right)^{2}+\left(\frac{1}{\sqrt{3}} \frac{\Delta \gamma}{2}\right)^{2}}
$$

where $\Delta \varepsilon$ and $\Delta \gamma$ are, respectively the range of axial strain and engineering shear strain in an axial-torsional test of a thin-walled tubular specimen. In the multiaxial test, a proportional loading parameter, $\lambda$, is introduced as [23]

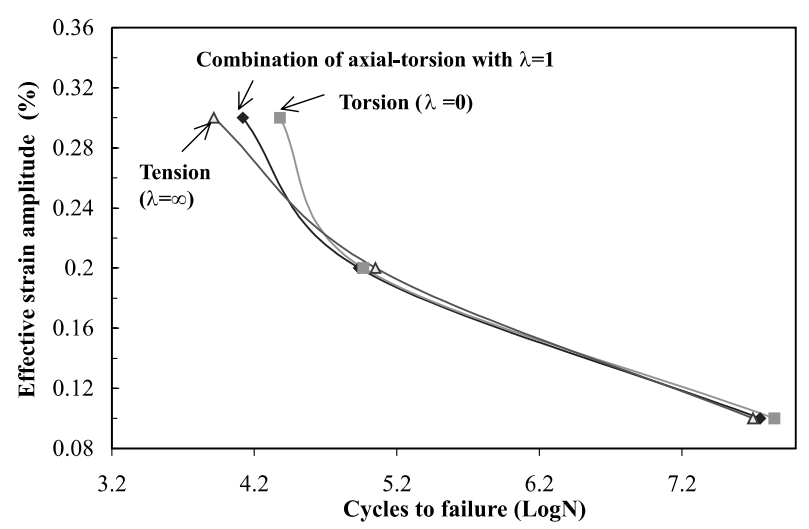

Fig. 5. Experimentally measured fatigue life versus effective strain amplitude for a cast A356-T6 Al alloy under completely reversed cyclic multiaxial loading of pure torsion, tension-compression, and a combination of tension-compression and torsion. 


$$
\lambda=\sqrt{3} \frac{\Delta \varepsilon}{\Delta \gamma}
$$

Multiaxial fatigue tests included completely reversed tension-compression $(\lambda=\infty)$, pure torsion $(\lambda=0)$, and a combination of tension-compression and torsion with $\lambda=1$. Effective strain amplitudes were imposed ranging from $0.1 \%$ to $0.3 \%$. The specimens had an outside diameter of $17.8 \mathrm{~mm}$, inside diameter of $14.0 \mathrm{~mm}$, and a gage length of $35.6 \mathrm{~mm}$. The fatigue tests were conducted using a MTS-809 Servo Hydraulic Axial-Torsional system. The effective strain rate at the mean diameter was $5 \times 10^{-3} \mathrm{~s}^{-1}$. Rate effects can be neglected.

Due to the low porosity level, strain-life curves shown in Fig. 5 exhibit little scatter. For relatively high strain amplitudes (above $0.2 \%$ ), distinct differences are measured between fatigue life obtained for tensioncompression, pure torsion, and their combination. However, when the effective strain (stress) amplitude is less than $0.2 \%$, little difference exists between the three loading conditions on the basis of the von Mises effective strain measure. These test results show that the fatigue strength at $10^{7}$ cycles is well correlated using the von Mises criterion for the low porosity A356-T6 Al alloy specimens.

\subsection{Computational elastoplastic micromechanical analysis}

To understand the experimental observations, a realistic microstructure representation of the low porosity A356-T6 Al alloy was obtained via a 2D optical image analysis and then meshed for the plane strain finite element calculations. More details of the finite element modeling technique and boundary conditions can be found in Ref. [3]. The FEM model uses an outer region and an inner region; the outer region is an homogeneous effective medium, while the inner region employs a detailed meshing of the heterogeneous microstructure. There were approximately 29,000 elements in the mesh with the smallest size being $0.5 \mu \mathrm{m}$. This microstructure idealization excludes pores. Fig. 6 shows the distribution of effective plastic strain in particle clusters under a completely reversed remote cyclic tension-compression strain amplitude of $0.2 \%$ along the $x$-direction. Since particles were assumed as linear elastic, all particle domains appear as white, i.e. zero plastic strain. From this figure, we see that all particles were intact. It was found that the local

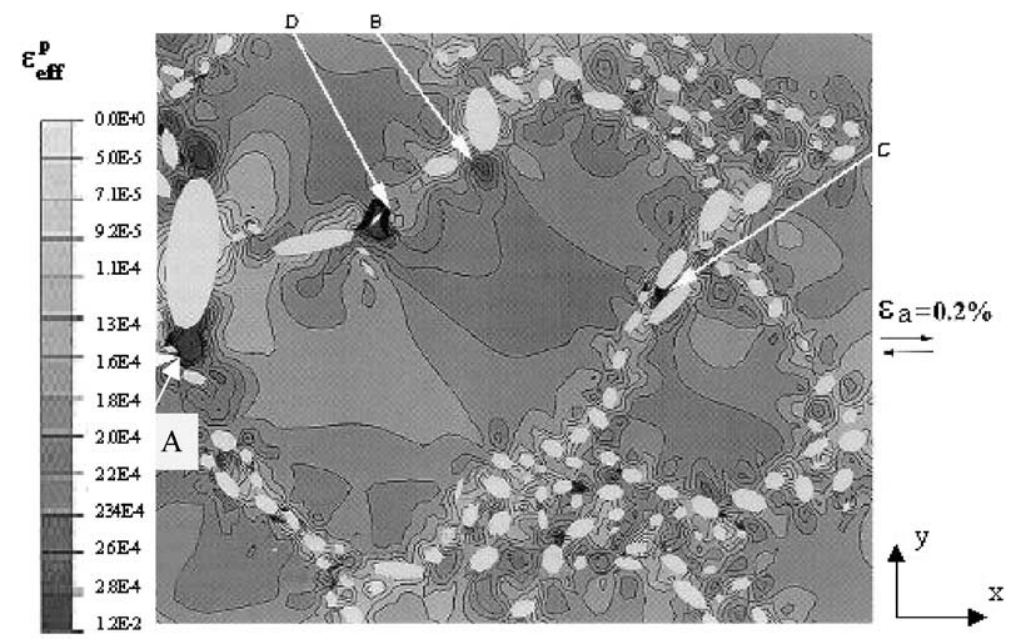

Fig. 6. Distribution of effective plastic strain, $\varepsilon_{\text {eff }}^{\mathrm{p}}$, within a realistic microstructure with intact particle/matrix interface for a cast A356T6 Al alloy $\left(R_{\varepsilon}=-1, \varepsilon_{\mathrm{a}}=0.2 \%\right.$. Boundary conditions: uniform cyclic displacement along the $x$-direction at the left side, simple support at the right side with $u_{x}=0$, simple support at the bottom side with $u_{y}=0$, and free at the top side). 


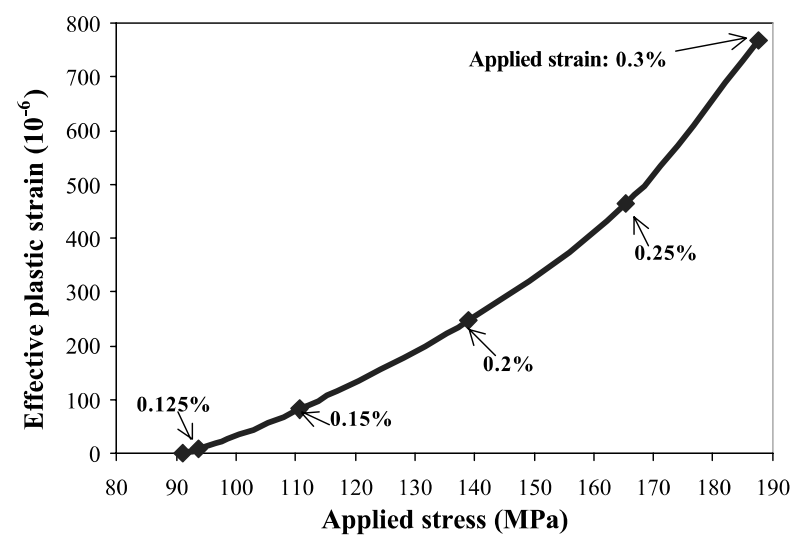

Fig. 7. Maximum local effective plastic strain as a function of applied stress from finite element simulations of a representative realistic microstructure of a cast A356-T6 alloy.

microplastic strain concentration occurred near the particle tips (site A and B), microcrack (site D), or between particles (site C). The maximum local microplastic strain was as high as $247 \mu \varepsilon$ for an applied, completely reversed total strain amplitude of $0.2 \%$. For this strain amplitude, the overall specimen response was essentially in the elastic range, and the corresponding stress amplitude is approximately $133 \mathrm{MPa}$.

Furthermore, numerical simulations were conducted for applied remote strain amplitudes of $0.1 \%$, $0.125 \%, 0.15 \%, 0.2 \%, 0.25 \%$, and $0.3 \%$. For each case, the corresponding maximum local microplastic strain was determined. However, for an applied strain amplitude of $0.1 \%$ (stress amplitude of about 74 $\mathrm{MPa}$ ), no plastic strain was found for the low porosity microstructure. The results obtained for the other five simulations are given in Fig. 7. The maximum local effective plastic strain, $\varepsilon_{\text {eff }}^{\mathrm{p}}=\left(2 / 3 \varepsilon_{i j}^{\mathrm{p}} \varepsilon_{i j}^{\mathrm{p}}\right)^{1 / 2}$, versus applied stress plot shows that as the applied strain amplitude decreased, the maximum local effective plastic strain decreased. By extrapolation, the local maximum microplastic strain approached zero for an applied strain amplitude of $0.122 \%$ (approximately $90 \mathrm{MPa}$ ).

From Figs. 6 and 7, it is observed that the maximum predicted strain amplitude below which local yielding within the microstructure (microplasticity) does not occur is close in value to that determined experimentally for a fatigue life of $10^{7}$ cycles. This corresponds to an applied stress amplitude of $90 \mathrm{MPa}$. From these calculations, it appears that there is a critical stress amplitude below which there is insufficient local plasticity within the microstructure to drive fatigue crack formation, or to propagate pre-existing small crack-like defects within a lifetime of practical interest. In practice, when fatigue test data are unavailable, generalized empirical factors are often used to determine the fatigue strength as a fraction of the static ultimate strength $S_{\mathrm{u}}$ [24]. These empirical factors are used mainly for steels. Insufficient data exist for casting aluminum alloys to use such empirical relations. The results in this section offer some promise for the potential of computational micromechanics performed on "realistic" microstructures to establish understanding of HCF fatigue limits. However, more work needs to be done to fully verify this consideration, including $\mathrm{HCF}$ experiments and 3D simulations.

\section{Parametric study of effects of particles on fatigue crack incubation}

Here we focus attention on determining the effects of particle size, spacing, aspect ratio, and particle clustering. An idealized particle cluster with four particles arranged in two rows and two columns is used as shown in Fig. 4. All calculations were conducted under completely reversed strain controlled loading 


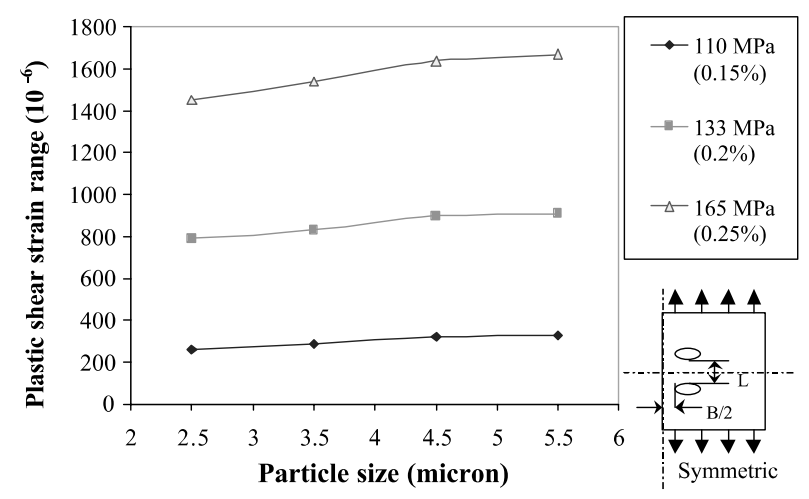

Fig. 8. Effect of particle size on the average (over a distance of $2 \mu \mathrm{m}$ along loading direction) maximum plastic shear strain range for applied stress amplitudes of 110,133 and $165 \mathrm{MPa}\left(R_{\varepsilon}=-1, D_{\max } / D_{\min }=1.65, L=10 \mu \mathrm{m}, B / L=1 ; \alpha=0.5, C=0.0211\right)$.

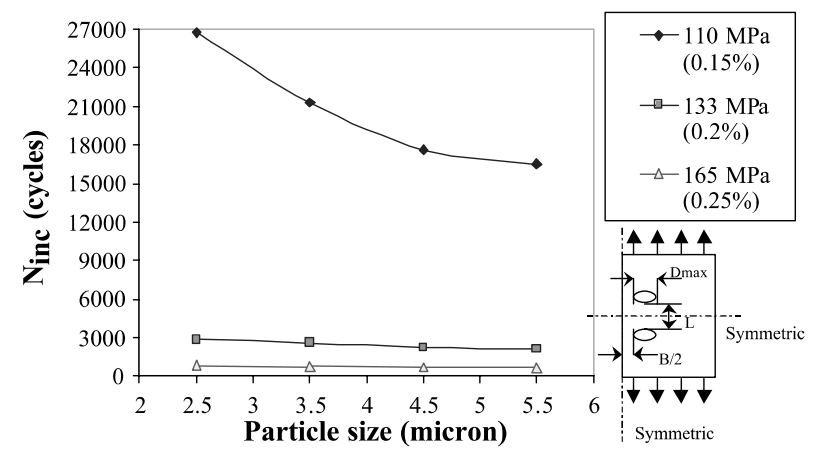

Fig. 9. Effect of particle size on fatigue crack incubation life for applied stress amplitudes of 110,133 and $165 \mathrm{MPa}\left(R_{\varepsilon}=-1\right.$, $\left.D_{\max } / D_{\min }=1.65, L=10 \mu \mathrm{m}, B / L=1 ; \alpha=0.5, C=0.0211\right)$.

conditions at strain amplitudes of $0.15 \%, 0.2 \%$ and $0.25 \%$. These simulations assumed perfectly intact particles without porosity. Figs. 8 and 9 show the effects of the particle size on the maximum range of plastic engineering shear strain, $\Delta \gamma_{\max }^{\mathrm{p}^{*}}$ and the number of cycles for crack incubation based on the local Coffin-Manson relation in Eq. (1) for the Al-rich matrix material. These results indicate that the maximum plastic shear strain range increases and the number of cycles for crack formation decreases with increasing particle size over the size range analyzed. The sensitivity of incubation fatigue life to particle size is much more pronounced at the lower stress amplitude of $110 \mathrm{MPa}$ than at stress amplitudes above the point of macroscopic yielding.

Figs. 10 and 11 show the effects of a particle clustering arrangements aligned along the loading direction. Please refer to Fig. 4 for geometric definitions. The results show that the maximum range of plastic shear strain increases quickly as the number of particles in a cluster increases from one to three, and then approaches a limit when more than three particles are involved. Accordingly, the number of cycles for fatigue crack incubation decreases with an increase of number of particles in a cluster. The sensitivity of fatigue crack incubation life to the number of particles is most pronounced at lower stress levels than that at stress levels above macroscopic yielding. This observation can perhaps be used to explain the observation of Plumtree and Schafer [5] that cracks originating at the silicon particles in the eutectic region generally form at triple points, since triple points involve stacking of particles. The effects of particle aspect ratio and normalized longitudinal particle spacing on the maximum range of plastic shear strain and $N_{\text {inc }}$ can also be 


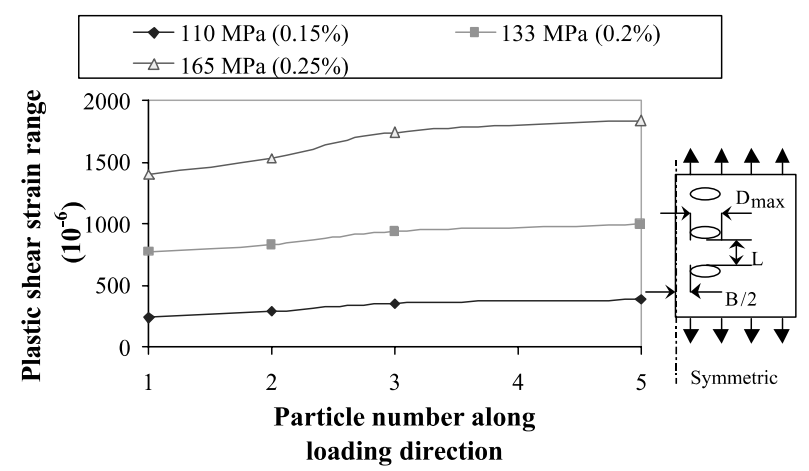

Fig. 10. Effect of number of particles in cluster (number of particles stacked parallel to the loading direction) on the average (over a distance of $2 \mu \mathrm{m}$ along loading direction) maximum plastic shear strain range for applied stress amplitudes of 110,133 and $165 \mathrm{MPa}$ $\left(R_{\varepsilon}=-1, D=7 \mu \mathrm{m}, D_{\max } / D_{\min }=1.65, L / D=0.85, B / L=1.43\right)$.

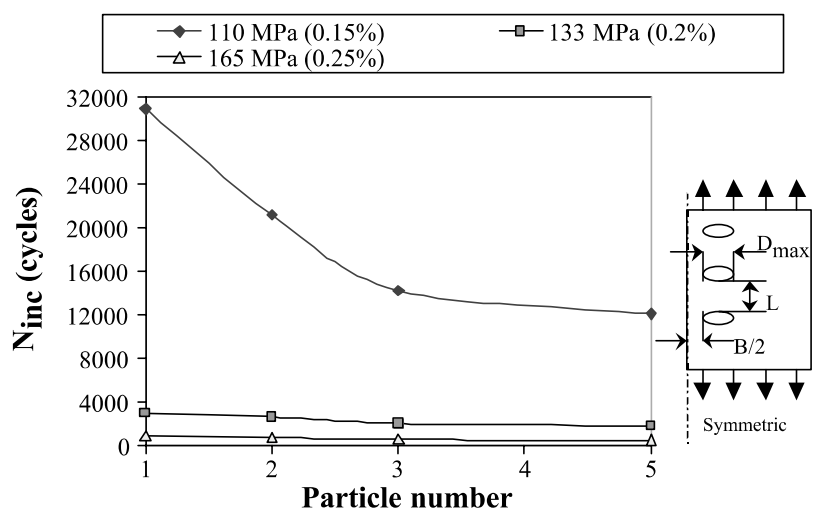

Fig. 11. Effect of number of particles in cluster (number of particles stacked parallel to the loading direction) on fatigue crack incubation life for applied stress amplitudes of 110,133 and $165 \mathrm{MPa}\left(R_{\varepsilon}=-1, D=7 \mu \mathrm{m}, D_{\max } / D_{\min }=1.65, L / D=0.85, B / L=1.43\right)$.

found, respectively, in Figs. 12-15. For a particle aspect ratio ranging from 1.0 to $1.65, N_{\text {inc }}$ is higher when the ratio is close to unity. Concerning effects of spacing between two particles along the loading direction, the results indicate that the maximum range of plastic shear strain is highest when the spacing between particles along the loading direction is on the order of the particle size. Particle aspect ratio effects, at least over the range considered, are less significant than the number of particles in the cluster or spacing effects, especially in the HCF regime.

\section{Parametric study of effects of pores on fatigue crack incubation}

One of the most deleterious flaws in a cast alloy is a large pore. In this section we examine effects of pores on crack incubation using both realistic pore microstructures and idealized geometries in finite element analyses. With the exception of analyses in Section 6.3, all pores are assumed to reside in the bulk with negligible interaction with the free surface. 


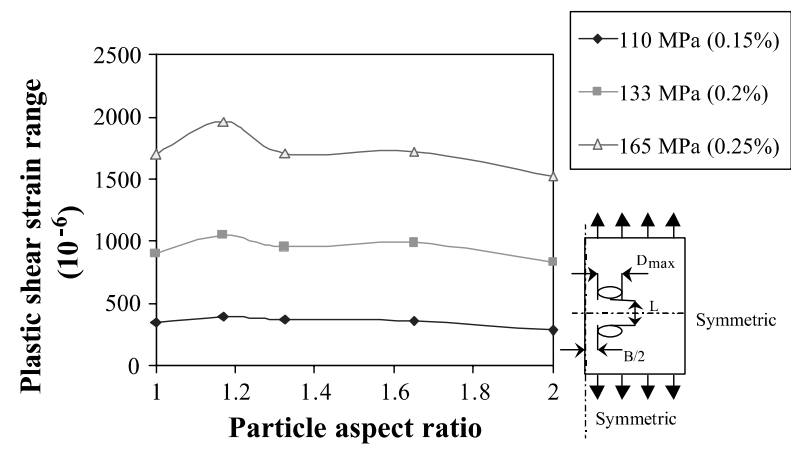

Fig. 12. Effect of particle aspect ratio on the average (over a distance of $2 \mu \mathrm{m}$ along loading direction) maximum plastic shear strain range for applied stress amplitudes of 110,133 and $165 \mathrm{MPa}\left(R_{\varepsilon}=-1, D=7 \mu \mathrm{m}, L / D=1.43, B / D=2.86\right)$.

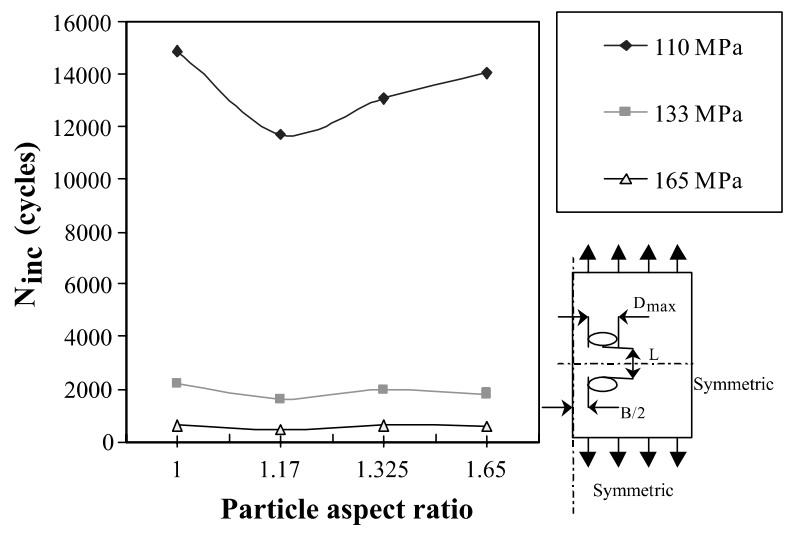

Fig. 13. Effect of particle aspect ratio on fatigue crack incubation life for stress amplitudes of 110,133 and $165 \mathrm{MPa}\left(R_{\varepsilon}=-1\right.$, $D=7 \mu \mathrm{m}, L / D=1.43, B / D=2.86)$.

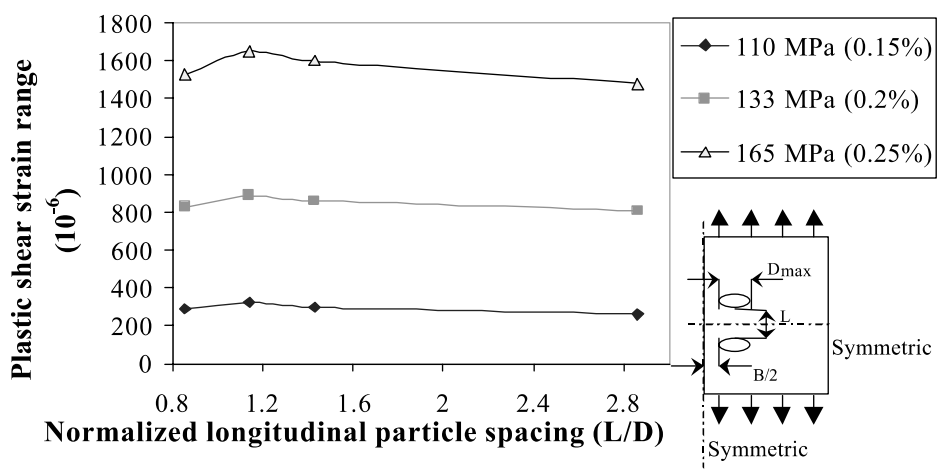

Fig. 14. Effect of normalized longitudinal particle spacing on the average (over a distance of $2 \mu \mathrm{m}$ along loading direction) maximum plastic shear strain range for applied stress amplitudes of 110,133 and $165 \mathrm{MPa}\left(R_{\varepsilon}=-1, D_{\max } / D_{\min }=1.65, D=7 \mu \mathrm{m}, B / D=2.86\right)$. 


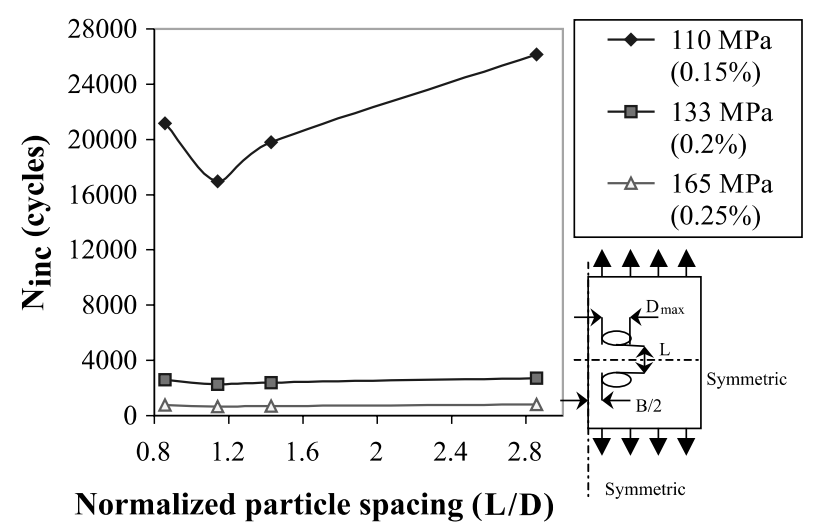

Fig. 15. Effect of normalized longitudinal particle spacing on fatigue crack incubation life for applied stress amplitudes of 110,133 and $165 \mathrm{MPa}\left(R_{\varepsilon}=-1, D_{\max } / D_{\min }=1.65, D=7 \mu \mathrm{m}, B / D=2.86\right)$.

\subsection{Realistic pore simulations}

We obtained results for distribution of the largest principal stress and effective plastic strain near a realistic pore of A356-T6 Al alloy taken from optical image analysis under completely reversed cyclic tension-compression. The maximum and minimum dimensions of the pore in this case are 205 and $131 \mu \mathrm{m}$. Over the perimeter of the pore, there are some arcs with small radii of curvature. The minimum local radius of curvature is about $3.5 \mu \mathrm{m}$. The maximum effective plastic strain and principal stress coincide with the arcs near the major axis that is perpendicular to the loading direction. As the radius of curvature decreases, the stress/strain concentration increases. This indicates that the local curvature of the pore has an influence on fatigue crack incubation. For a pore with an effective pore size of $55 \mu \mathrm{m}$ subjected to a strain amplitude of $0.2 \%$, the maximum plastic strain amplitude was $1260 \mu \varepsilon$ and the stress concentration factor was approximately two. When the pore size was increased from 55 to $168 \mu \mathrm{m}$, the stress concentration factor doubled to approximately four and the maximum effective plastic strain increased from 1260 to $21,500 \mu \varepsilon$.

\subsection{Idealized microstructure: parametric studies for bulk pores}

To investigate the relative effects of size, spacing, local curvature, and clustering of pores on fatigue crack incubation, an idealized pore cluster with four pores arranged in the pattern of Fig. 4 was subjected to remote completely reversed tension-compression strain amplitudes of $0.15 \%, 0.2 \%$ and $0.25 \%$. It is assumed that there is no interaction with the free surface. To simulate pore interactions, symmetry boundary conditions are used so that the four pores can be considered as a periodic array along both longitudinal and transverse directions. It should be noted that for randomly distributed pore clusters, the fatigue life is mainly controlled by clusters with the largest pore sizes.

We obtained distributions of the maximum principal stress and the maximum plastic shear strain in the pore cluster of four pores. The pore size is $D_{\max }=500 \mu \mathrm{m}$; the pore aspect ratio is two, and the minimum local radius of curvature is $35 \mu \mathrm{m}$. For an applied strain amplitude of $0.2 \%$ (133 MPa), the maximum stress is $324 \mathrm{MPa}$, and the maximum range of plastic shear strain is $7960 \mu \varepsilon$. The stress concentration factor is 2.43 , and the ratio of the maximum range of plastic shear strain to the applied strain amplitude is 3.98. Within the size range of pores and particles investigated here, the maximum range of plastic shear strain of the matrix within pore clusters is about 15-30 times higher than that of the matrix in particle clusters. 


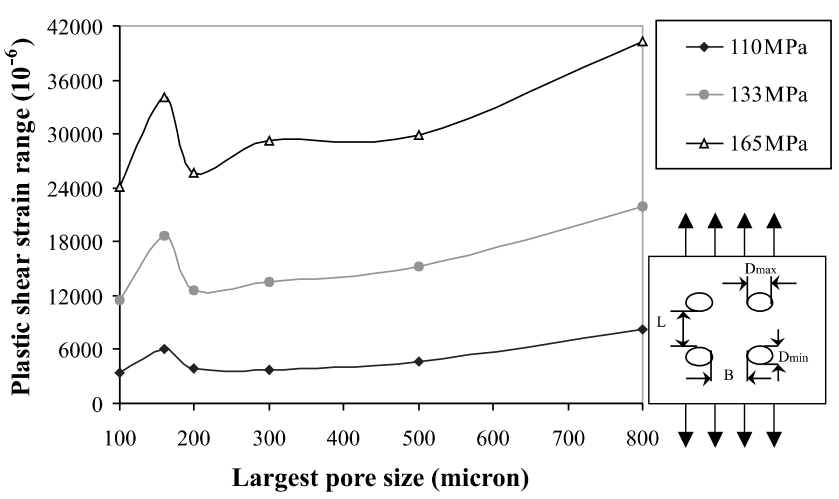

Fig. 16. Effect of pore size on the average (over a distance of $20 \mu \mathrm{m}$ perpendicular to the loading direction) maximum plastic shear strain range for a contrived cluster of four pores for applied stress amplitudes of 110,133 and $165 \mathrm{MPa}\left(R_{\varepsilon}=-1, D_{\max } / D_{\min }=2\right.$, $B=L=720 \mu \mathrm{m})$.

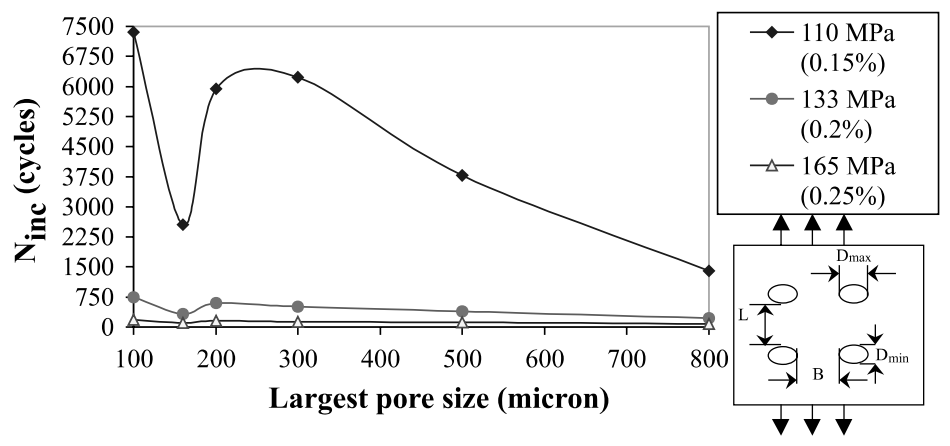

Fig. 17. Effect of pore size on fatigue crack incubation life for a contrived cluster of four pores for applied stress amplitudes of 110,133 and $165 \mathrm{MPa}\left(R=-1, D_{\max } / D_{\min }=2, B=L=720 \mu \mathrm{m}, \alpha=0.5464, C=0.0622\right)$.

Figs. 16 and 17 show the effects of the largest pore size on the maximum range of plastic shear strain and the number of cycles for crack incubation. The effects of pore spacing and the minimum local curvature radius of the pore cluster are shown, respectively, in Figs. 18-21. By examining these figures, we can draw several conclusions:

(a) Fig. 16 shows the relationship between $\Delta \gamma_{\max }^{\mathrm{p} *}$ and the largest pore size, $D_{\max }$, for the fixed pore spacing of $720 \mu \mathrm{m}$. The larger the pore size, the larger the $\Delta \gamma_{\max }^{\mathrm{p} *}$, resulting in a lower $N_{\text {inc }}$ for crack incubation (see Fig. 17). One interesting phenomenon is that when the pore size reaches about $180 \mu \mathrm{m}$, there is a local maximum of $\Delta \gamma_{\max }^{\mathrm{p} *}$. This exceptionally large value of $\Delta \gamma_{\max }^{\mathrm{p} *}$ at $D_{\max }=180 \mu \mathrm{m}$ occurs when the pore spacing is about four times the largest pore size. This local maximum of $\Delta \gamma_{\max }^{\mathrm{p} *}$ is caused by interactions between slip bands of pores which are displaced along the diagonal of the four-pore clusters shown in Fig. 4. Their slip bands were approximately along the same directions of $45^{\circ}$.

(b) Figs. 18 and 19 show that the smaller the pore spacing, the higher the $\Delta \gamma_{\max }^{\mathrm{p} *}$ and the lower the $N_{\text {inc }}$. This arises because small pore spacing induces more severe pore interactions, resulting in more plastic shear strain intensification. There is a competition between the effects of pore size and spacing size on plastic strain intensification. From the discussion of (a) above, it may be reasonable to assume that the exceptional local maximum can occur for other pore spacing and the corresponding diameter of the pore 


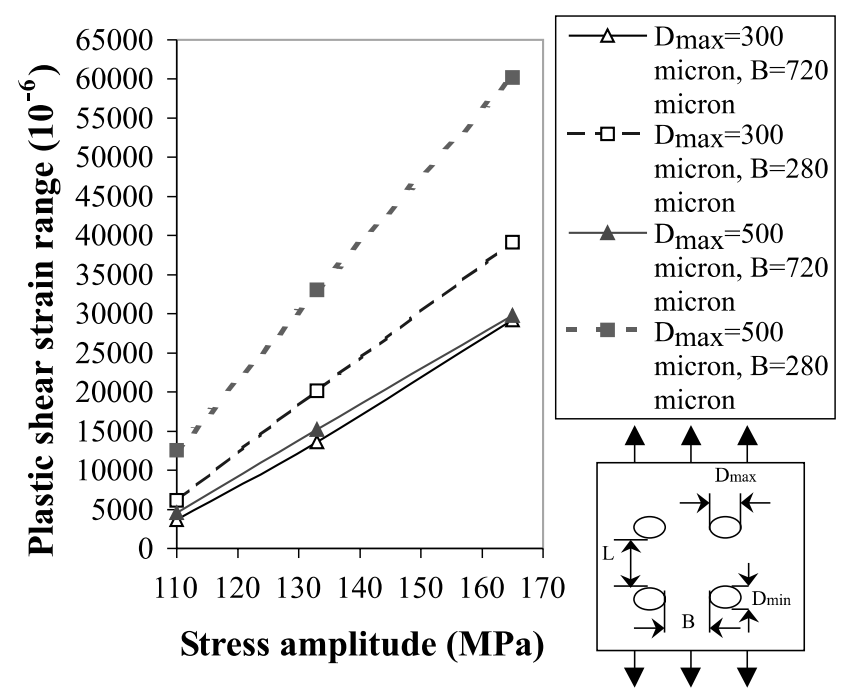

Fig. 18. Effect of pore spacing for a contrived cluster of four pores on the maximum plastic shear strain range for pore sizes of $D_{\max }=300$ and $500 \mu \mathrm{m}$ as a function of the applied stress amplitude $\left(R_{\varepsilon}=-1, D_{\max } / D_{\min }=2, B / L=1, \alpha^{\prime}=0.5464, C^{\prime}=0.0622\right)$.

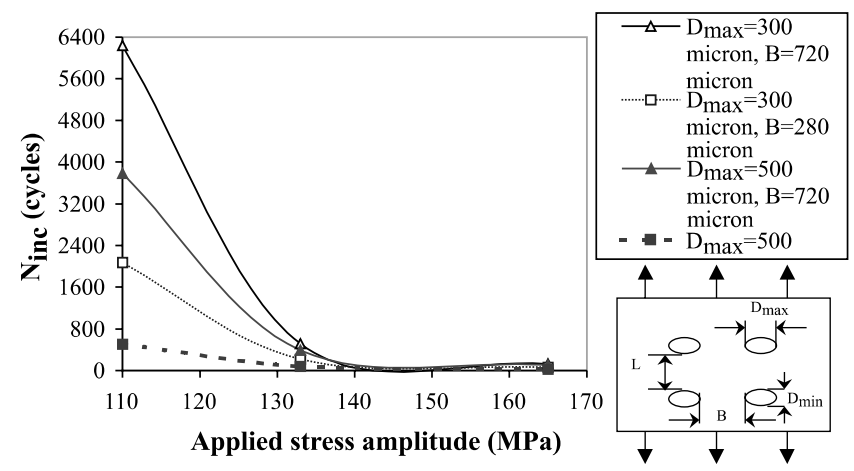

Fig. 19. Effect of pore spacing for a contrived cluster of four pores on fatigue crack incubation life for pore sizes of $D_{\max }=300$ and 500 $\mu \mathrm{m}$ as a function of the applied stress amplitude $\left(R_{\varepsilon}=-1, D_{\max } / D_{\min }=2, B / L=1, \alpha^{\prime}=0.5464, C^{\prime}=0.0622\right)$.

depends on the spacing. Such considerations may be useful in avoiding unfavorable morphologies of pore clusters. However, it should be confirmed through more calculations and carefully designed experiments.

(c) The relative sensitivity of plastic shear strain, $\Delta \gamma_{\max }^{\mathrm{p} *}$, to increase of pore size is higher at lower stress amplitudes than at higher stress amplitudes above the point of macroscopic yielding. For example, for a completely reversed, cyclic tension-compression loading, the computed $\Delta \gamma_{\max }^{\mathrm{p} *}$ of $8260 \mu \varepsilon$ for the $800 \mu \mathrm{m}$ pore is 2.36 times larger than that for the $100 \mu \mathrm{m}$ pore under the stress amplitude of 110 $\mathrm{MPa}$; this ratio decreases to 1.9 when the stress amplitude increased to $165 \mathrm{MPa}$, with the $\Delta \gamma_{\max }^{\mathrm{p} *}$ of $21,900 \mu \varepsilon$ for the $800 \mathrm{~mm}$ pore. When the local curvature radius of the pore decreased from 15 to 7 $\mu \mathrm{m}$, the local plastic strain range increased rapidly. The corresponding $N_{\text {inc }}$ is small; it is only 100 and 7 cycles for the stress amplitudes of 133 and $165 \mathrm{MPa}$, respectively. For a local radius of curvature within the range of $15-30 \mu \mathrm{m}, N_{\mathrm{inc}}$ is approximately proportional to the local radius of curvature. 


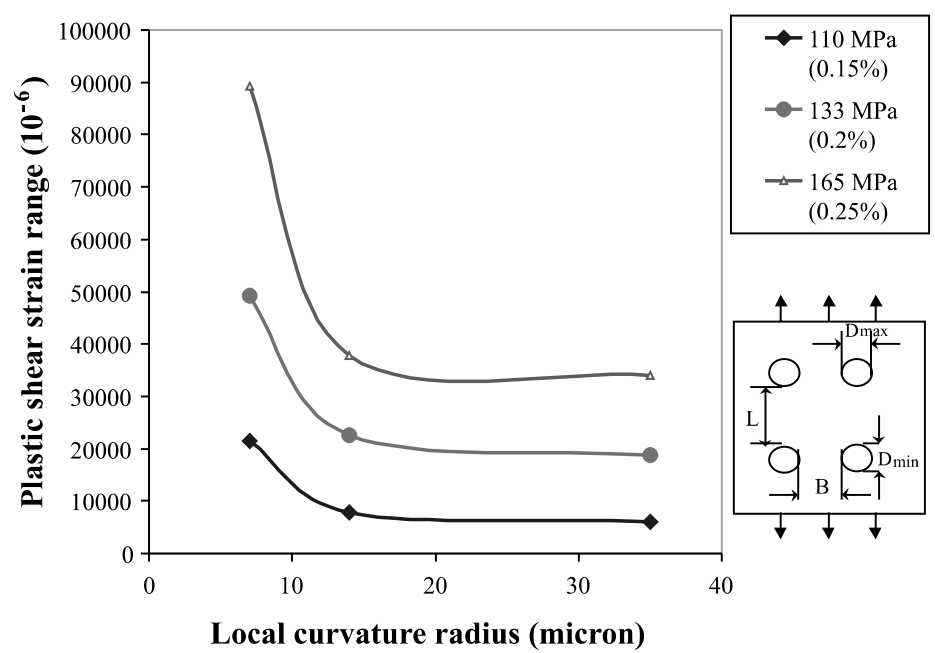

Fig. 20. Effect of the minimum radius of curvature for a contrived cluster of four pores on the average (over a distance of $20 \mu \mathrm{m}$ perpendicular to the loading direction) maximum plastic shear strain range for applied stress amplitudes of 110,133 and $165 \mathrm{MPa}$ $\left(D_{\max } / D_{\min }=2, D_{\max }=150 \mu \mathrm{m}, R_{\varepsilon}=-1\right)$.

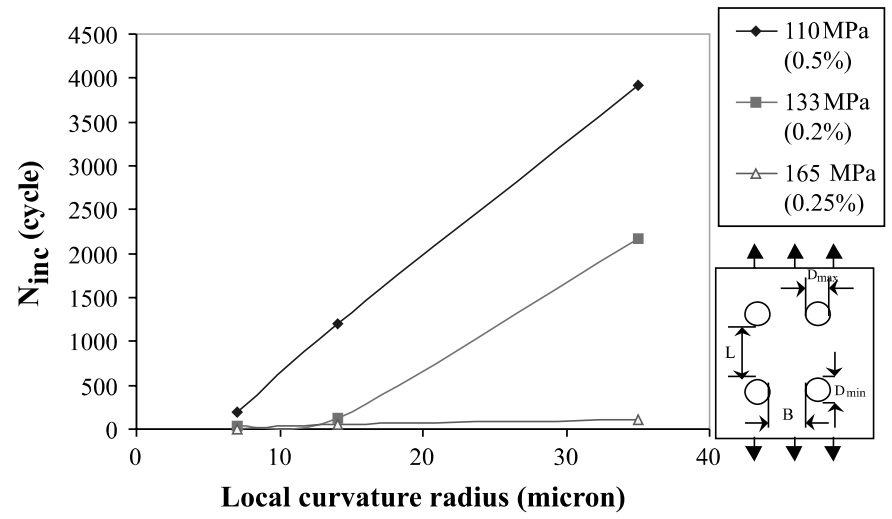

Fig. 21. Effect of the minimum radius of curvature for a contrived cluster of four pores on fatigue crack incubation life for applied stress amplitudes of 110,133 and $165 \mathrm{MPa}\left(D_{\max } / D_{\min }=2, D_{\max }=150 \mu \mathrm{m}, R_{\varepsilon}=-1\right)$.

\subsection{Pores near the free surface}

For pores near the free surface, Fig. 1 shows an intensification of cyclic plastic strain (slip) between the pores and the free surface under cyclic loading. The maximum range of plastic shear strain, the cycles for crack incubation, and the distance between the free surface and the pore extreme under completely reversed tension-compression cyclic loading are shown in Figs. 22 and 23. The results show that $\Delta \gamma_{\max }^{\mathrm{p} *}$ decreases and $N_{\text {inc }}$ increases rapidly as the pore moves away from near the free surface to the inside of the material. After the distance from the surface increases to about the same order of the pore's largest dimension, the decay of $\Delta \gamma_{\max }^{\mathrm{p} *}$ with distance from the free surface ceases, indicating a negligible effect of the free surface on fatigue crack incubation when the pore is deep inside of the material. Fig. 22 shows that as the applied stress amplitude increases, there are more severe effects of the free surface on the $\Delta \gamma_{\max }^{\mathrm{p} *}$ compared to those at lower stress amplitude. Constraint effects of the material on plastic deformation are much higher for an 


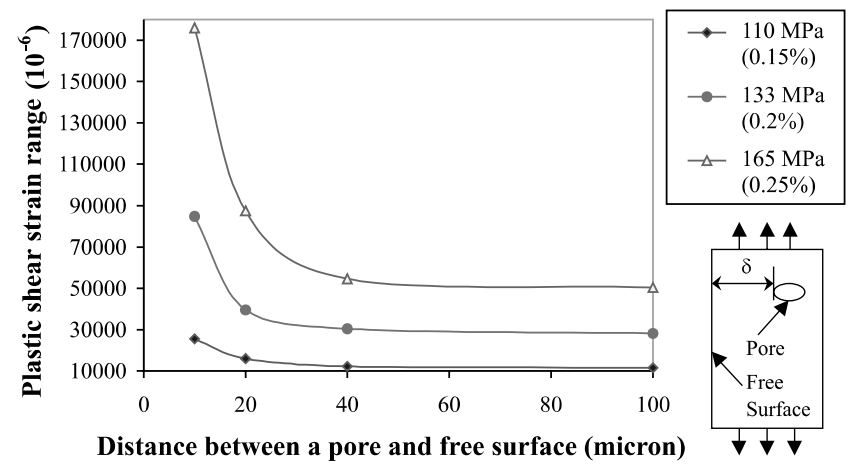

Fig. 22. Effect of the distance between a pore and a free surface on the average (over a distance of $7.5 \mu \mathrm{m}$ perpendicular to the loading direction) maximum plastic shear strain range for applied stress amplitudes of 110,133 and $165 \mathrm{MPa}\left(R_{\varepsilon}=-1, D_{\max } / D_{\min }=2\right.$, $\left.D_{\max }=40 \mu \mathrm{m}\right)$.

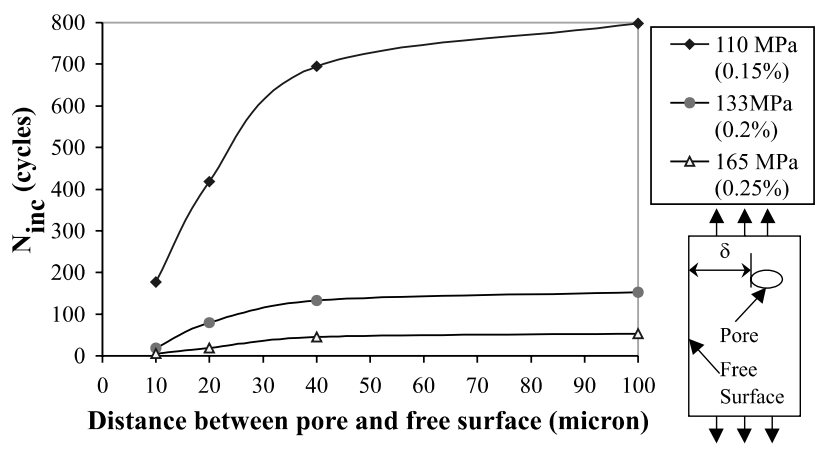

Fig. 23. Effect of the distance between a pore and free surface on fatigue crack incubation life for applied stress levels of 110 , 133 and $165 \mathrm{MPa}\left(D_{\max } / D_{\min }=2, D_{\max }=40 \mu \mathrm{m}, R_{\varepsilon}=-1, \alpha^{\prime}=0.5464, C^{\prime}=0.0622\right)$.

interior pore than for the pores that is close to the free surface. In fact, the values of $\Delta \gamma_{\max }^{\mathrm{p} *}$ for a pore cluster is much smaller, in general, than those obtained for a single pore near a free surface. For example, the smallest $\Delta \gamma_{\max }^{\mathrm{p} *}$ for a stress amplitude of $165 \mathrm{MPa}$ is about $50,000 \mu \varepsilon$ for a single pore with a size of $40 \mu \mathrm{m}$ which has a distance of $10 \mu \mathrm{m}$ from the free surface; however, the value of $\Delta \gamma_{\max }^{\mathrm{p} *}$ for a bulk pore with a size of $100 \mu \mathrm{m}$ at the same stress level is only about $25,000 \mu \varepsilon$.

\section{Conclusions}

(1) Fatigue cracks that initiate at intersections of silicon particle clusters, large pores, oxide films, and sites near fractured and debonded particles appear to arise from local microstructure-induced plastic shear straining/slip intensification. The cyclic plastic strains from these finite element simulations were used to develop local Coffin-Manson type laws for fatigue crack incubation. Hence, the number of cycles spent on fatigue crack incubation was quantified, elucidated, and not related to classical crack initiation concepts. Crack initiation concepts typically contain a significant amount of microstructurally small crack propagation, which is not included in our crack incubation formulation. Also, the minimum element size in the finite element simulations was selected to assure proper physical averaging at the intensification region. 
(2) Multiaxial (axial-torsional) fatigue tests were conducted for tubular specimens for a cast A356-T6 A1 alloy microstructure with an extremely low overall volume fraction of porosity. Given that SEM revealed no evidence of crack formation at pores [22], representative microstructures of these low porosity specimens obtained from optical images were used for a 2D finite element analysis, idealized as zero porosity. One interesting result was that when the remote strain (stress) amplitude for completely reversed loading was reduced to $0.122 \%$ (about $90 \mathrm{MPa}$ ), the calculated maximum microplastic strain approached zero. This FEM-determined maximum strain amplitude was found to be close in value to the experimentally determined fatigue strength at $10^{7}$ cycles. For the multiaxial tests, a Mises effective strain criterion was used and validated for correlating results for strain amplitudes below the macroscopic cyclic yield point.

(3) Effects of particle size, spacing, aspect ratio, and particle clustering were examined, as well as the effects of pore size, spacing, the distance of a pore from surface, local curvature, and clustering. Stress amplitudes were also varied (three levels) in order to determine the driving force for fatigue crack incubation. A general result observed was that sensitivity to microstructure is particularly strong for applied stress amplitudes below the macroscopic yield point, i.e., the HCF regime. While the parametric results are helpful to identify trends related to size and morphology of pores/particles on the range of local plastic strain concentration and fatigue crack incubation, the calculations are based on a highly idealized $2 \mathrm{D}$ analyses. Therefore, caution should be exercised in applying these results directly to life prediction models.

\section{Acknowledgements}

This work was performed as a collaborative effort between Georgia Tech and Sandia National Laboratories under US DOE contract DE-AC04-94AL85000, supported by USCAR Program. Several other groups are to be recognized for their involvement in this program. Daimler-Chrysler Corporation and Alcan Inc. provided the A356-T6 cast aluminum alloy plates and Westmoreland Mechanical Research and Testing machined and tested specimens. Richard J. Osborne and Donald E. Penrod are acknowledged for significant guidance and support of this program. Contributions from General Motors, Ford Motor Company, and American Foundry Society are appreciated. The first author also thanks the National Science Foundation of China through the contract of 19772096.

\section{References}

[1] McDowell DL, Gall KA, Horstemeyer MF, Fan J. Microstructure-based fatigue modeling of cast A356-T6 alloy. Engng Fract Mech, in press.

[2] Brockenbrough JR, Hinkle AJ, Magnusen PE, Bucci RJ. Microstructurally based model of fatigue and growth. FAA/NASA International Symposium on Advanced Structural Integrity Methods for Airframe Durability and Damage Tolerance, NASA Conference Publication 3274, Part 1, 1994. p. 71-84.

[3] Fan J, McDowell DL, Horstemeyer MF, Gall K. Computational micromechanics analysis of cyclic crack tip behavior for microstructurally small cracks in dual-phase alloys. Engng Fract Mech 2001;68:1687-706.

[4] McDowell DL. Stress state dependence of the cyclic ratchetting behavior of two rail steels. Int J Plast 1995;11:397-421.

[5] Plumtree A, Schafer S. Initiation and short crack behavior in aluminum alloy castings. In: Miller KJ, de los Rios Rios, editors. The behaviour of short fatigue cracks, EGF Pub 1. London: Mechanical Engineering Publications; 1986. p. $215-27$.

[6] Lee FT, Major JF, Samuel FH. Effect of silicon particles on the fatigue crack growth characteristics of $\mathrm{Al}-12 \mathrm{wt} \% \mathrm{Si}-$ 0.35wt.\%Mg-(0-0.02)wt.\%Sr casting alloys. Metall Mater Trans A 1995;26A:1553-70.

[7] Gall K, Horstemeyer M, McDowell DL, Fan J. On the driving force for fatigue crack formation from inclusions and voids in a cast A356 aluminum alloy. Int J Fract 2001;108:207-33.

[8] Gurland J, Plateau J. The mechanism of ductile rupture of metals containing inclusions. Trans ASM 1963;56:442-54. 
[9] Yeh J-W, Liu W-P. The cracking mechanism of silicon particles in an aluminum alloy. Metall Mater Trans 1996;A27:3558.

[10] Wang QG, Apelian D. Microstructural effects on the fatigue properties of aluminum castings. In: Tiryakioglu M, Campbell J, editors. Advances in aluminum casting technology. ASM International; 1998. p. 217-24.

[11] Gall K, Yang N, Horstemeyer M, McDowell DL, Fan J. The debonding and fracture of si particles during the fatigue of a cast AlSi alloy. Metall Mater Trans A 1999;30:3079-88.

[12] Ting JC, Lawrence FV. Modeling the long-life fatigue behavior of a cast aluminum alloy. Fat Frac Engng Mat Struc 1993;16(6):631-47.

[13] Dabayeh AA, Berube AJ, Topper TH. An experimental study of the effect of a flaw at a notch root on the fatigue life of cast Al A319. Int J Fat 1998;20:517-30.

[14] Couper MJ, Neeson AE, Griffith JR. Casting defects and the fatigue behaviour of an aluminum casting alloy. Fat Fract Mater Struct 1990;13(3):213-27.

[15] Venkataraman G, Chung YM, Mura T. Application of minimum energy formation in a multiple slip band model for fatigue--I. Calculation of slip band spacing. Acta Metall Mater 1991;39(11):2621-9.

[16] Venkataraman G, Chung YM, Mura T. Application of minimum energy formation in a multiple slip band model for fatigue-II. Crack nucleation and derivation of a generalized Coffin-Manson law. Acta Metall Mater 1991;39(11):2631-8.

[17] Kato M, Onake S, Mori T, Mura T. Scripta Metall 1984;18:1323.

[18] Socie DF. Critical plane approaches for multiaxial fatigue damage assessment. In: McDowell DL, Ellis R, editors. Advances in multiaxial fatigue, ASTM STP 1191, 1993. p. 7-36.

[19] McDowell DL. In: ASM Handbook 19 on Fatigue and Fracture. ASM International; 1996. p. $263-73$.

[20] Shackelford JF. Introduction to materials science for engineers. fifth ed. Prentice Hall; 2000.

[21] ABAQUS, Hibbit, Karlsson, and Sorenson, Inc., Pawtucket, RI, 1998.

[22] Gall K, Yang N, Horstemeyer M, McDowell DL, Fan J. The influence of modified intermetallics and Si particles on fatigue crack paths in a cast A356 Al alloy. Fat Fract Engng Mater Struct 2000;23:159-72.

[23] McDowell DL. A two-surface model for transient nonproportional cyclic plasticity. ASME J Appl Mech 1985;52:298-308.

[24] Juvinall RC, Marshek KM. Fundamentals of machine component design. third ed. New York: John Wiley \& Sons Inc.; 2000. 